\title{
May the Central Nervous System Be Fogged by the Cytokine Storm in COVID-19?: an Appraisal
}

\author{
Yasin Hasan Balcioglu ${ }^{1}$ (D) Umit Haluk Yesilkaya ${ }^{1} \cdot$ Hasan $_{\text {Gokcay }}{ }^{2} \cdot$ Simge Seren Kirlioglu $^{1}$
}

Received: 27 May 2020 / Accepted: 5 June 2020 / Published online: 11 June 2020

(C) Springer Science+Business Media, LLC, part of Springer Nature 2020

Dear editor:

We recently read with interest the article by Acharya et al. entitled "SARS-CoV-2 Infection Leads to Neurological Dysfunction" (Acharya et al. 2020). They have reviewed current literature on the neurological complications and manifestations of COVID-19, however, they were able to identify only a very limited number of studies. In fact, in our latest paper, we have provided a deeper insight on the pathophysiology of the central nervous system involvement of COVID19 and discussed that neuropsychiatric manifestations related COVID-19 might be associated with the involvement of both direct viral transmission and neuroimmune response (Yesilkaya and Balcioglu 2020). Latter includes heightened systemic proinflammatory response which is closely associated with so-called cytokine storm, a well-known phenomenon led by SARS-CoV-2 infection.

As viral replication progressively decreases during the latter days of infection, the pathogenesis of COVID-19 has been thought to be predominantly related to an aggressive inflammatory response. Thus, the intense release of proinflammatory cytokines (viz cytokine storm) in the host leads to diffuse alveolar damage, severe hypoxemia, and promotes the development of fatal secondary sepsis (Serrano-Castro et al. 2020). Even though the cytokine storm has been a well-defined condition in many viral infections including influenza and coronavirus (Liu et al. 2016), our appreciation of its effects on the central nervous system (CNS) has remained limited.

The primary targets for SARS COV-2 are respiratory epithelial cells and alveolar macrophages. Following the

Yasin Hasan Balcioglu

yhasanbalcioglu@gmail.com

1 Department of Psychiatry, Bakirkoy Prof Mazhar Osman Training and Research Hospital for Psychiatry, Neurology, and Neurosurgery, Istanbul, Turkey

2 Department of Psychiatry, Bagcilar Training and Research Hospital, University of Health Sciences, Istanbul, Turkey infection of respiratory cells, rapid production and release of many chemokines and cytokines initiate. Macrophages are activated by such proinflammatory chemokines and cytokines, and other key components of the innate immune system, dendritic cells, lead to a more extensive immune response that initiates the cytokine storm. Circulating chemokines attract more inflammatory cells to make them migrate from blood vessels into the site of inflammation, and these cells release additional chemokines/cytokines which amplify cytokine storm (Liu et al. 2016). Although TNF- $\alpha$, IL-1, and IL-6 are primary cytokines of the acute immune response and the interferons are key cytokines for antiviral immune response, the excessive response of these proinflammatory mediators have been considered major triggers of sepsis and multiorgan failure (Unal et al. 2020). On the other hand, the CNS might be the first affected organ due to a compromised blood-brain barrier which is closely associated with increased circulating levels of cytokines. These cytokines induce the production of reactive oxygen species through a stimulation of astrocytes and glial cells (Meneses et al. 2019).

Reactive oxygen species and peripheral cytokines activate two different types of microglia; microglia-1 (M1) and microglia-2 (M2). While M1 involve in inflammatory processes in the CNS, M2 are responsible for the antiinflammatory processes. Thus, M1 activation results in an increase of proinflammatory cytokines and M2 initiate the astrogliosis in order to CNS protection. All of these opposite reactions induced by SARS-COV-2 may cause a CNS dysfunction that becomes apparent as encephalopathy. Another pathologic reaction related to SARS-CoV-2 might involve in sensorial (olfactory) nerves. Olfactory nerves are activated by pathogen-associated molecular patterns (PAMPs) and inflammatory cytokines. The viral transmission in the olfactory bulb leads a neuroinflammatory response through an excessive release of cytokines to protect the CNS. Therefore, anosmia, an initial symptom of COVID-10, might be a result of such neuroinflammatory response (Meneses et al. 2019). 
Despite the arguments identified above, our knowledge is still limited to what extent such neuroinflammatory reactions does SARS-COV-2 associate with. We would like to emphasize the importance of long term follow-up and neuropsychiatric monitoring should be performed in patients with COVID-19. Psychoneuroimmunological characteristics of COVID-19, which seem to be key for the neurological involvement of the disease still needs to be elucidated, indeed.

\section{Compliance with Ethical Standards}

Conflict of Interest The authors declared no potential conflicts of interest with respect to the research, authorship, and/or publication of this article.

\section{References}

Acharya A, Kevadiya BD, Gendelman HE, Byrareddy SN (2020) SARS$\mathrm{CoV}-2$ infection leads to neurological dysfunction. J NeuroImmune Pharmacol:1-7. https://doi.org/10.1007/s11481-020-09924-9
Liu Q, Zhou Y, Yang Z (2016) The cytokine storm of severe influenza and development of immunomodulatory therapy. Cell Mol Immunol 13:3-10

Meneses G, Cárdenas G, Espinosa A et al (2019) Sepsis: developing new alternatives to reduce neuroinflammation and attenuate brain injury. Ann N Y Acad Sci 1437:43-56

Serrano-Castro PJ, Estivill-Torrús G, Cabezudo-García P et al (2020) Impact of SARS-CoV-2 infection on neurodegenerative and neuropsychiatric diseases: a delayed pandemic? Neurol (English Ed). https://doi.org/10.1016/j.nrleng.2020.04.002

Unal G, Turan B, Balcioglu YH (2020) Immunopharmacological management of COVID-19: potential therapeutic role of valproic acid. Med Hypotheses:109891. https://doi.org/10.1016/j.mehy.2020. 109891

Yesilkaya UH, Balcioglu YH (2020) Neuroimmune correlates of the nervous system involvement of COVID-19: a commentary. J Clin Neurosci. https://doi.org/10.1016/j.jocn.2020.05.056

Publisher's Note Springer Nature remains neutral with regard to jurisdictional claims in published maps and institutional affiliations. 